\title{
Core structure of flavonoids precursor as an antihyperglycemic and antihyperlipidemic agent: an in vivo study in rats
}

\author{
Mahmoud Najafian', Azadeh Ebrahim-Habibi², Parichehreh Yaghmaei ${ }^{\circledR}$, Kazem Parivar ${ }^{1}$ and \\ Bagher Larijani²
}

Science and Research Branch, Islamic Azad University, Tehran, Iran; Endocrinology and Metabolism Research Center, Tehran University of Medical Sciences, Tehran, Iran

\begin{abstract}
trans-Chalcone is the core structure of naringenin chalcone, located halfway in the biosynthesis pathway of flavonoids. Flavonoids have been reported as mammalian alpha-amylase inhibitors, a property which could be useful in the management of postprandial hyperglycemia in diabetes and related disorders. As a mammalian alphaamylase inhibitor in vitro, the putative beneficial effect of trans-chalcone on diabetes was tested in a streptozotocin-induced rat model of diabetes type 1, and the results analyzed with commonly used statistical methods. Significant reduction of blood glucose levels and beneficial effect on dyslipidemia were observed in diabetic rats, as well as reduction of disturbing consequences of diabetes such as high urine volume and water intake. trans-Chalcone was observed to have a weight loss-inductive effect, alongside with a reduction in food intake, which is suggestive of a therapeutic potential of this compound in overweight and obese patients.
\end{abstract}

Keywords: trans-chalcone, diabetes, benzylideneacetophenone, alpha-amylase inhibitor, hyperglycemia, hyperlipidemia

Received: 14 June, 2010; revised: 10 August, 2010; accepted: 22 September, 2010; available on-line: 09 November, 2010

\section{INTRODUCTION}

According to the International Diabetes Federation (IDF) (http://www.diabetesatlas.org), the global prevalence of diabetes is predicted to grow from $6.6 \%$ in 2010 to $7.8 \%$ in 2030 , and the global prevalence of impaired glucose tolerance from $7.9 \%$ in 2010 to $8.4 \%$ in 2030. Translation of these percentages to numbers would give 439 million people suffering from diabetes in 2030, and 472 million with impaired glucose tolerance. Diabetes is cited in the IDF report as being the fourth or fifth leading cause of death in most highincome countries and an epidemic disease in economically developing and newly industrialized nations. Its chronic characteristic leads to progressive complications which affect life quality, and as such needs to be attended to in preventive and therapeutic means.

Among the multiple molecular targets that are aimed at in diabetes, hydrolase enzymes acting in carbohydrate digestion are currently gaining more attention, since postprandial hyperglycemia is known to be an important factor in diabetic complications (Ceriello, 2005; Rebolledo \& Actis Dato, 2005). The first inhibitor of $\alpha$-glucosidase to be commercialized, acarbose, has been shown to be safe and maintain its effect in the long-term (Rosak \& Mertes, 2009). It may also re- duce the risk of cardiovascular disease by influencing insulin and pro-insulin as risk factors of cardiovascular dysfunction (Rosak \& Mertes, 2009). The acarbose effect in the control of postprandial hyperglycemia is suggested to make this compound a potential preventive aid in metabolic syndrome complications (Yamagishi et al., 2005). Acarbose is also an inhibitor of $\alpha$-amylase, although in micromolar concentrations (Yoon \& Robyt, 2003), and other well-known inhibitors of $\alpha$-amylase have been shown to be effective in the control of postprandial hyperglycemia, while devoid of the diarrhea side effect (Boivin et al., 1988; Lankisch et al., 1998) associated with acarbose (Godbout \& Chiasson, 2007).

Classical inhibitors of mammalian $\alpha$-amylase include protein and carbohydrate-like structures, the latter mainly based on acarbose (Machius et al., 1996). In recent years, small molecules have also been studied in this regard, and among these flavonoids have been reported to exhibit such activity (Kim et al., 2000; Tadera et al., 2006; Lo Piparo et al., 2008).

Flavonoids are a widely studied group of natural polyphenolic compounds which have been attributed a variety of therapeutical properties such as: antiatherosclerotic, anti-inflammatory, antitumor, antithrombogenic, antiosteoporotic, and antiviral (Nijveldt et al., 2001). Flavonoid-rich fractions of plants have also been reported to be effective as antihyperglycemic and antihyperlipidemic agents in animal models of diabetes (Aslan et al., 2007; Li et al., 2007; Sharma et al., 2008). trans-Chalcone is a precursor of flavonoids in plants (Verhoeyen et al., 2002; Ferrer et al., 2008). Based on the similarity of its structure to that of the flavonoids (trans-chalcone could be considered a simplified flavonoid), its inhibitory effect toward mammalian $\alpha$-amylase was investigated. The compound was found to be effective in the micromolar range (Najafian et al., 2010). The current study was therefore designed to observe whether this compound was also effective in vivo. Rats with streptozotocin-induced diabetes (usually considered as a model of type 1 diabetes mellitus) were treated with four doses of the compound, which showed actual benefit in lowering serum glucose and lipid levels.

\footnotetext{
¿e-mail: yaghmaei_p@srbiau.ac.ir
}

Abbreviations: D, diabetic rats; DO, diabetic control group; DCh2, DCh8, DCh16, and DCh32, diabetic rats receiving chalcone $2,8,16$, or $32 \mathrm{mg} / \mathrm{kg}$ respectively; ND, non-diabetic rats; NDO, non-diabetic control rats; NDCh2, NDCh8, NDCh16, NDCh32, non-diabetic rats receiving chalcone respectively, 2, 8, 16, or $32 \mathrm{mg} / \mathrm{kg}$; HDL, highdensity lipoproteins; LDL, low-density lipoproteins; STZ, streptozotocin; TG, triglycerides; VLDL, very low-density lipoproteins 


\section{MATERIAL AND METHODS}

Chemicals. trans-Chalcone (benzylideneacetophenone) was from Sigma Chemical Co. (St. Louis, MO, USA) and streptozotocin from Pharmacia \& Upjohn (Kalamazoo, MI, USA).

Animals. Male adult Wistar rats (2.5 months) weighting $200 \pm 15 \mathrm{~g}$ were used in these experiments. The animals were housed as six rats per cage at room temperature $\left(22-24^{\circ} \mathrm{C}\right)$ with lights on from 08:00 to 20:00 h. Rats received standard rodent diet: maintenance diet Letica, Panlab S.L. (Barcelona, Spain); 61.4\% (w/w) carbohydrate (100\% starch), $3.9 \%$ fibre, $15.1 \%$ protein and $2.7 \%$ fat, and tap water. Food and water were ad libitum.

Diabetes induction. Diabetic condition was induced by the use of a single dose of streptozotocin (STZ) (70 $\mathrm{mg} / \mathrm{kg}$ body weight) dissolved in a citrate buffer $(0.1$ $\mathrm{mol} / \mathrm{l}), \mathrm{pH}$ 4.5. STZ was administered intraperitoneally on the first day of experiment according to existing protocols (Portha et al., 1974; 1989; Tormo et al., 2004), and the blood glucose levels were measured after 2 days. The ethical aspect of the experimental protocol was approved by the ethical committee of the Science and Research Branch of Islamic Azad University (Tehran, Iran).

Treatment with trans-chalcone. Rats were divided into two classes (non-diabetic rats (ND) and diabetic rats (D)) and each class divided into five groups $(n=6)$, as defined below:

(I) Non-diabetic control group (NDO): rats in this group received grape seed oil (O) orally for 24 days through a gastric cannula in a single dose $(0.5 \mathrm{ml})$ at 8:30 a.m. (II) Diabetic control group (DO): rats in this group received grape seed oil (O) as group I. (III) Non-diabetic groups receiving trans-chalcone (NDCh): rats in these groups received trans-chalcone at 2, 8, 16, $32 \mathrm{mg} / \mathrm{kg}$ body weight (respectively, NDCh2, NDCh8, NDCh16, NDCh32) dissolved in grape seed oil, orally for 24 days through a gastric cannula in a single dose $(0.5 \mathrm{ml})$ at 8:30 a.m. (IV) Diabetic groups receiving trans-chalcone $(\mathrm{DCh})$ : rats in these groups received transchalcone at 2, 8, 16, $32 \mathrm{mg} / \mathrm{kg}$ body weight (respectively, DCh2, DCh8, DCh16, and DCh32) as above. Throughout the manuscript, chalcone doses of $2,8,16,32 \mathrm{mg} /$ $\mathrm{kg}$ body weight are shown as $\mathrm{Ch} 2, \mathrm{Ch} 8$, etc.

Measured parameters. As rats were fed at night ad libitum, ingestion of food and water, as well as urine volume was measured every morning at 9:00 a.m. Blood glucose levels were measured in $2 \mu \mathrm{l}$ blood samples ex-

A

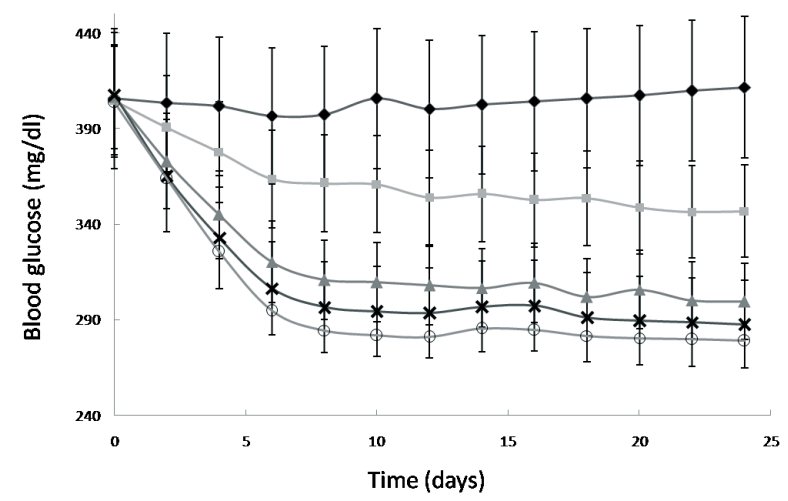

tracted from the tail of the animal every two days on mornings at 9:00 a.m. with a glucometer (One Touch Profile, Life Scan). Serum insulin levels were determined three times during the experiment period, that is at day one, day 12 and day 24, with the use of insulin kits (DRG, France), using double-antibody enzymelinked immunosorbent assay (ELISA). At the end of experiment period, the body weight of all animals was measured and they were sacrificed under light ether anesthesia. The abdomen was cut open, and blood collected from heart. Blood samples were placed on ice and centrifuged within $15 \mathrm{~min}$ after collection at $3000 \times g$ for five minutes. Serum was stored at $-20{ }^{\circ} \mathrm{C}$ for less than 1 week before subsequent analyses. Standard biochemical methods were used to measure cholesterol (Deeg \& Ziegenhorn, 1983), triglycerides (Cole et al., 1997), highdensity lipoproteins (HDL) (Wiebe \& Warnick, 1997), low-density lipoproteins (LDL) (Bachorik, 1997) concentrations, and $\alpha$-amylase activity (Kruse-Jarres et al., 1989). Estimates of very low density lipoprotein (VLDL) were calculated from the formula VLDL-C = triacylglycerol $/ 5$ (Friedewald et al., 1972)

Statistical analysis. Standard deviation was calculated in all cases and results expressed as mean \pm SD. The data were analyzed by one-sample Kolmogrov-Smirnov test and then by the Levene's test. One way analysis of variance (ANOVA) followed by Tukey's post hoc test for multiple comparisons were used to compare difference between experimental groups. The criterion for statistical significant was $P<0.05$.

\section{RESULTS AND DISCUSSION}

\section{Glucose}

Administration of STZ resulted in high levels $(405.6 \pm 41.9 \mathrm{mg} / \mathrm{dl})$ of blood glucose after 2 days in the treated group in contrast with the non-diabetic control group in which blood glucose levels were in the range of $104.8 \pm 11.9 \mathrm{mg} / \mathrm{dl}$.

In our previous in vitro experiments, trans-chalcone, being virtually insoluble in aqueous medium was dissolved in dimethyl sulfoxide (Najafian et al., 2010). Given the hydrophobic character of the compound, it was necessary to choose a suitable hydrophobic vehicle for its administration to rats, hence the choice of grape seed oil. Grape seed ethanolic extract has been suggested to

B

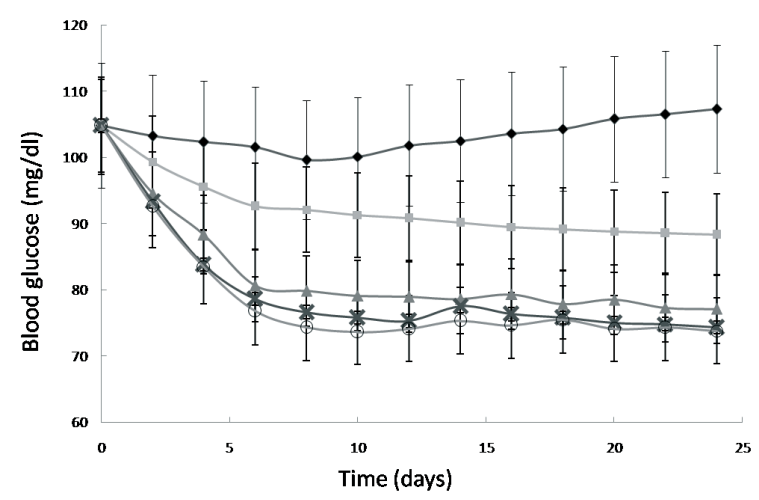

Figure 1. Effect of trans-chalcone on blood glucose in diabetic and non-diabetic rats Rats were given grape seed oil $(\bullet)$ or chalcone dissolved in oil of doses $2(\boldsymbol{\square}), 8(\mathbf{\Delta}), 16(\mathbf{x})$, and $32(\bigcirc) \mathrm{mg}$ per kg body weight for 24 days. Values are means \pm S.D. (A) Diabetic rats. (B) Non-diabetic rats. 
(A)

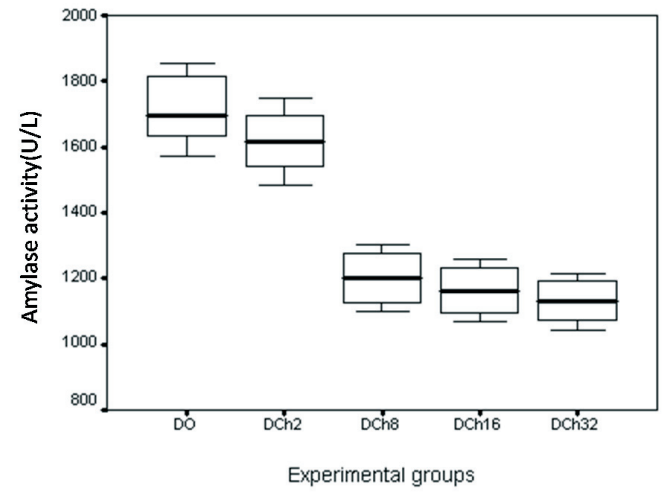

(B)

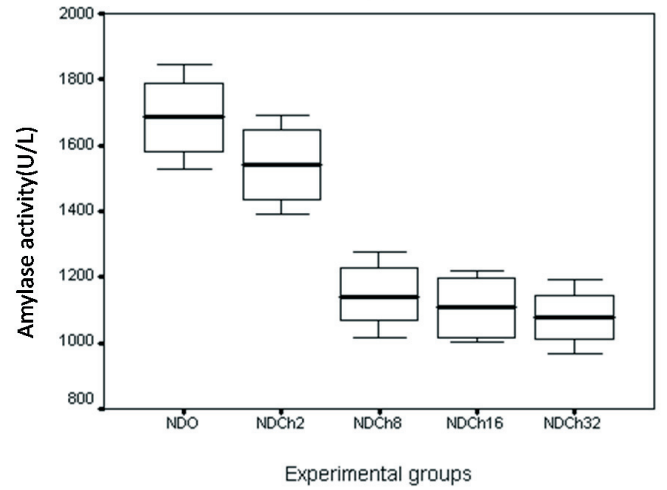

Figure 2. Effect of trans-chalcone treatment on a-amylase activity in diabetic and non-diabetic rats

(A) Diabetic rats. Using one way ANOVA with Tukey-Kramer test at $P<0.05$, the following results were obtained for the data: DCh8 is significantly different from DO and DCh2; DCh16 is significantly different from DO and DCh2, DCh32 is significantly different from DO and DCh2. (B) Non-diabetic rats. Using one way ANOVA with Tukey-Kramer test at $P<0.05$ following results were obtained: NDCh8 is significantly different from NDO and NDCh2; NDCh16 is significantly different from NDO and NDCh2; NDCh32 is significantly different from NDO and NDCh2.

be beneficial in a rat model of type 2 diabetes (Hwang et al., 2009), and grape seed oil content analysis has shown the presence of phytosterol (in concentrations between 5179 and $5480 \mathrm{mg} / \mathrm{kg}$ ) (Navas, 2009). However, the main component of grape seed "oil" seems to be linoleic acid, as observed in different varieties (Tangolar et al., 2007; Navas, 2009). In the current study, $0.5 \mathrm{ml}$ daily administered grape oil was found to be devoid of any effect on blood glucose levels of both diabetic and normal rats. In fact, these two groups showed unchanged blood glucose levels: in the non-diabetic control group (NDO) and in the diabetic control group (DO) blood glucose remained constant throughout the experimental period at about $104.8-107.3 \mathrm{mg} / \mathrm{dl}$ and $405.6-411.6 \mathrm{mg} / \mathrm{dl} \mathrm{respec}-$ tively (Fig. 1).

A decrease in glycemia was observed from day 2 after trans-chalcone administration at all the doses used. In each group this decrease occurred rapidly until day 6 and the blood glucose levels reached then were maintained until the end of the treatment (day 24). The effect appears to be dose-dependent, but there was no significant difference between the two doses of 16 and $32 \mathrm{mg} / \mathrm{kg}$ (Fig. 1). An interesting finding was that blood glucose levels were also decreased in the non-diabetic rats taking chalcone (Fig. 1B).

In this study, animals were not subjected to fasting before taking blood samples, thus the $\alpha$-amylase inhibitory property of trans-chalcone could have a role in the observed effect on the blood glucose levels. In a recent report, an effect of trans-chalcone and some of its derivatives has been shown on transient hyperglycemia induced by oral glucose gavage in normal rats. In that case, some chalcone derivatives were found to act in a similar manner as insulin (Alberton et al., 2008), suggesting the possibility of multiple targeting for these compounds.

\section{a-Amylase}

trans-Chalcone was found to be a competitive inhibitor of mammalian $\alpha$-amylase in vitro (Najafian et al., 2010); but given the complexity of in vivo systems, it could not be assumed to have the same effect when administered orally in a living organism. This is why the serum $\alpha$-amylase activity was also measured in this study (Fig. 2 ). Interestingly, $\alpha$-amylase activities of the diabetic and non-diabetic groups are comparable, as is the decrease in this activity, which was found to be significantly higher for the Ch8, Ch16 and Ch32 doses in comparison with the Ch2 dose while the difference between the three mentioned doses was not significant. This is possibly indicative of a saturation in the inhibitory effect of transchalcone toward $\alpha$-amylase. At any rate, this experiment shows that at least part of this compound's effect is exerted through inhibition of $\alpha$-amylase in vivo.

\section{Insulin}

Serum insulin levels of diabetic and non-diabetic rats sampled three times during the experiment are shown in Fig. 3. After STZ administration, serum insulin levels were significantly reduced in diabetic rats compared with non-diabetic rats, that is, $2.45 \pm 0.26$ vs. $3.85 \pm 0.41$ $\mathrm{ng} / \mathrm{ml}(P<0.01)$. In both control groups of diabetic and non-diabetic rats administered grape seed oil alone, the serum insulin levels remained nearly constant throughout the experiment. On the other hand, in both the diabetic and non-diabetic groups, given chalcone was administrated, halfway through the experiment (day 12), the serum insulin levels were significantly reduced. For example in DCh2 and NDCh2 a reduction was observed from $2.45 \pm 0.26$ to $1.61 \pm 0.18 \mathrm{ng} / \mathrm{ml}(P<0.01)$ and from $3.85 \pm 0.41$ to $2.95 \pm 0.18 \mathrm{ng} / \mathrm{ml}(P<0.01)$, respectively. In all groups this decline in serum insulin level was observed to be dose-dependent of chalcone from $2 \mathrm{mg} / \mathrm{kg}$ to $16 \mathrm{mg} / \mathrm{kg}$, while there was no significant difference between Ch16 and Ch32. From day 12 of experiment, until the last day (day 24) the serum insulin levels increased slightly.

It is interesting to note a similar observation made about the effect of a well studied food product containing an $\alpha$-amylase inhibitor, namely kidney bean (Phaseolus vulgaris), on rats. In that study, insulin plasma levels decreased when rats were fed kidney beans, while the amount of mRNAs for the insulin receptor and GLUT4 increased in a specific muscle type (Knott et al., 1992). The effect observed in the current study must be associated with the presence of chalcone and is independent of the pathological condition of diabetic rats, since it is also observed in non-diabetic rats, although the effect is less pronounced. Considering the fact that some chalcone derivatives act similarly to insulin-like compounds (Al- 

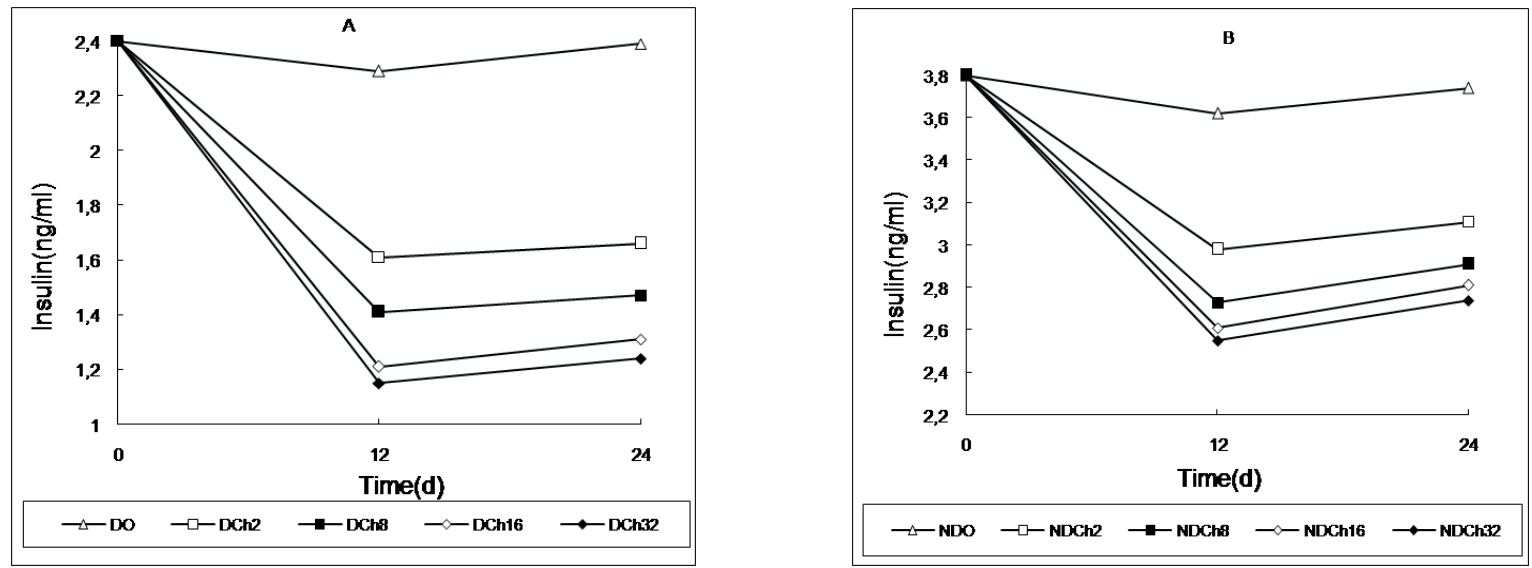

Figure 3. Effect of trans-chalcone treatment on plasma insulin levels in rats (A) Diabetic rats. (B) Non-diabetic rats.

Table 1. Effect of chalcone treatment in serum lipids of streptozotocin-induced diabetic rats

Data are expressed as mean \pm S.D. for six rats. a, significantly different from diabetic control group (DO); $b$, significantly different from DCh2; c, significantly different from DCh8; d, significantly different from DCh16 using one way ANOVA with Tukey-Kramer test at $P<0.05$.

\begin{tabular}{lcccccc}
\hline Serum level $(\mathrm{mg} / \mathrm{dl})$ & NDO & DO & DCh2 & DCh8 & DCh16 & DCh32 \\
\hline Cholesterol & $85.5 \pm 11.5$ & $125.5 \pm 17.2$ & $103.8 \pm 14.9$ & $95.5 \pm 15.1^{\mathrm{a}}$ & $93.3 \pm 17.3^{\mathrm{a}}$ & $95.1 \pm 15.6^{\mathrm{a}}$ \\
Triglycerides & $63.5 \pm 8.4$ & $119.6 \pm 18.9$ & $89.5 \pm 14.2$ & $76.6 \pm 10.7^{\mathrm{a}}$ & $78.3 \pm 12.6^{\mathrm{a}}$ & $275.5 \pm 34.4^{\mathrm{a}, \mathrm{b}, \mathrm{c}, \mathrm{d}}$ \\
VLDL-C & $12.7 \pm 1.7$ & $23.9 \pm 3.8$ & $17.9 \pm 2.8$ & $15.3 \pm 2.1^{\mathrm{a}}$ & $15.6 \pm 2.5^{\mathrm{a}}$ & $55.1 \pm 6.9^{\mathrm{a}, \mathrm{b}, \mathrm{c}, \mathrm{d}}$ \\
LDL-C & $22.5 \pm 4.6$ & $41.6 \pm 9.2$ & $27.6 \pm 4.6^{\mathrm{a}}$ & $22.3 \pm 5.4^{\mathrm{a}}$ & $25.6 \pm 6.0^{\mathrm{a}}$ & $25.5 \pm 5.7^{\mathrm{a}}$ \\
HDL-C & $44.5 \pm 9.6$ & $23.3 \pm 5.8$ & $34.5 \pm 7.3$ & $38.0 \pm 7.8^{\mathrm{a}}$ & $41.6 \pm 8.2^{\mathrm{a}}$ & $40.1 \pm 8.4^{\mathrm{a}}$ \\
\hline
\end{tabular}

berton et al., 2008) it could be suggested that the effect observed on plasma insulin could be a result of interactions between trans-chalcone and insulin receptors, possibly leading to down-regulation of insulin secretion. This is of course a pure speculation and needs further evidence.

\section{Lipids}

Dyslipidemia is observed as a consequence of both type 1 (Verges, 2009) and type 2 (Gosavi et al., 2006) diabetes, and could contribute to an increased incidence of cardiovascular diseases if untreated. However, it is usually observed that when type 1 diabetic patients have well-controlled glycemic levels, trigycerides (TG) and low density lipoproteins (LDL) become normal or slightly decrease, while a slight increase in high density lipoproteins (HDL) could also occur (Verges, 2009). In the current experiment, blood lipid levels of diabetic rats were also found to be elevated (Table 1). In the diabetic control group, in contrast to the non-diabetic control group, a marked elevation was observed in the concentration of serum cholesterol $(47 \%)$, TG $(88 \%)$, very low density lipoproteins (VLDL) (88\%), and LDL (85\%), while the concentration of HDL was reduced by $48 \%$. This lipid
(A)

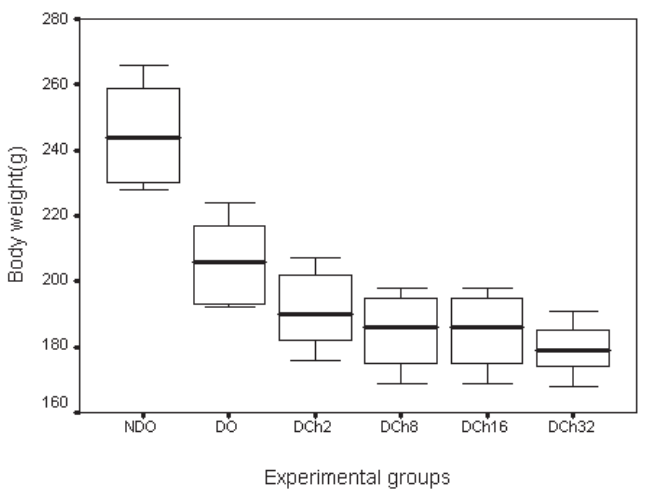

(B)

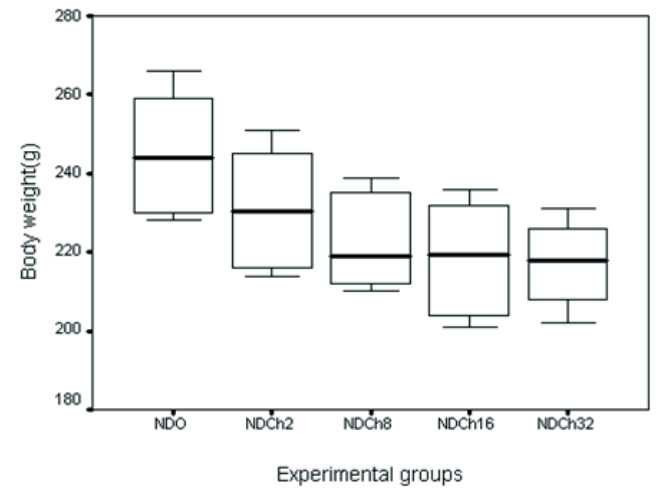

Figure 4. Effect of trans-chalcone treatment on body weight in diabetic and non-diabetic rats

(A) Diabetic rats. Using one way ANOVA with Tukey-Kramer test at $P<0.05$ following results were obtained for the data: DO is significantly different from NDO; DCh2 is significantly different from NDO; DCh8 is significantly different from NDO and DO; DCh16 is significantly different from NDO and DO; DCh32 is significantly different from NDO and DO. (B) Non-diabetic rats. Using one way ANOVA with TukeyKramer test at $P<0.05$ NDCh16 was found to be significantly different from NDO and NDCh32 significantly different from NDO. 
(A)

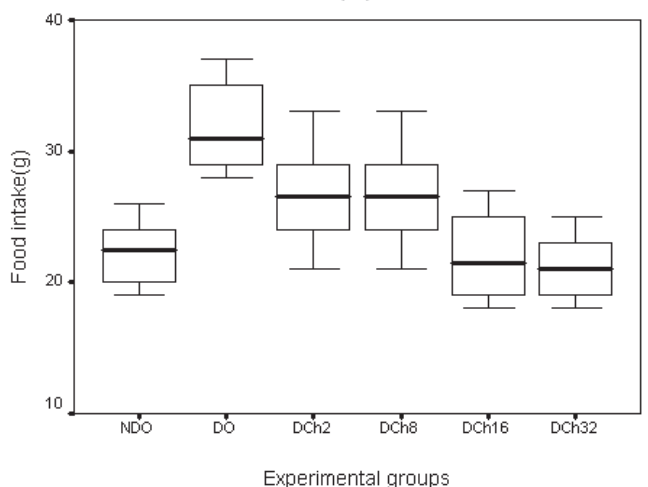

(B)

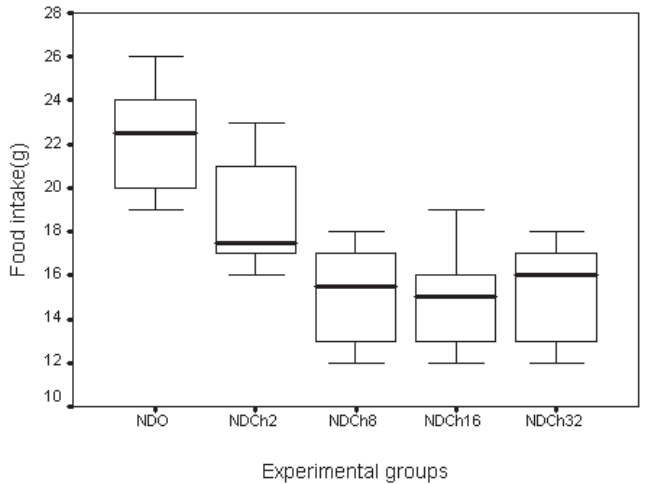

Figure 5. Effect of trans-chalcone treatment on food intake in diabetic and non-diabetic rats

(A) Diabetic rats. Using one way ANOVA with Tukey-Kramer test at $P<0.05$ following results were obtained for the data: DO is significantly different from NDO; DCh8 is significantly different from DO; DCh16 is significantly different from DO; DCh32 is significantly different from DO. (B) Non-diabetic rats. Using one way ANOVA with Tukey-Kramer test at $P<0.05$, NDCh8 was found to be significantly different from NDO; NDCh16 significantly different from NDO, and NDCh32 significantly different from NDO.

profile was remarkably improved after administration of chalcone, which is in accordance with the other effects of this compound observed in this study. It is interesting to note that the three doses Ch8, Ch16 and Ch32 had a similar effect on cholesterol, LDL and HDL concentration, and that Ch8 and Ch16 had a similar effect on triglycerides and VLDL concentration. On the other hand, the $\mathrm{Ch} 32$ dose acted in a reverse manner, and resulted in elevated levels of triglycerides and VLDL (more than two-fold), which suggests that this higher dose of chalcone would interfere in the lipid metabolism in some other way than what is observed for the lower doses.

As chalcone affected the blood glucose levels and insulin of normal rats, blood lipid levels of non-diabetic rats were also modified upon its administration ( $\mathrm{Ta}$ ble 2). It could be observed that in the experimental groups, the use of all doses (from 2 to $32 \mathrm{mg} / \mathrm{kg}$ ) had a similar reducing effect on the concentrations of cholesterol, triglycerides, VLDL, and LDL, while no significant effect was found on HDL. This effect on lipid profile is slightly different from the effect on blood glucose, possibly because it would be a more indirect result of chalcone interactions with its putative targets in vivo. It should be noted that the $32 \mathrm{mg} / \mathrm{kg}$ dose had no deleterious effect on the lipid profile, in contrast to its effect in diabetic rats.

\section{Body weight}

Use of chalcone was found to induce weight loss in both diabetic and non-diabetic rats. Results of body weight measurements done at day 24 are shown in Fig. 4. As a known consequence of type 1 diabetes, the body weight in the diabetic control group was significantly reduced in comparison with the non-diabetic control group $(206.3 \pm 13.1$ vs. $245.1 \pm 15.8, \quad P<0.01)$. When treated with chalcone, a dose-dependent body weight loss was observed in both diabetic and nondiabetic rats, although it was similar for the Ch16 and Ch32 doses. Weight loss has been suggested to be desirable in both type 1 and type 2 diabetic patients. Although this is more frequently a problem in patients with type 2 diabetes, since this condition is more likely to be associated with overweightness, unhealthy weight loss practices have also been found in youth with type 1 diabetes. As insulin intake is known to induce body weight increase, skipping insulin doses could occur in type 1 diabetic youth trying to lose weight (Lawrence et al., 2008). In this regard, the therapeutic potential of a compound able to lower glucose levels while also inducing weight loss would be interesting. This effect of chalcone could be related to its $\alpha$-amylase inhibitory property, or some kind of appetite-suppressing effect (as shown in the next section, chalcone treatment results in lower food intake).

\section{Food intake}

The effect of chalcone on food intake could be suggested to be part of its interaction with multiple targets. As shown in Fig. 5, food intake was significantly increased in the diabetic control group (DO) compared with the non-diabetic control group (NDO) $(31.8 \pm 3.5 \mathrm{~g}$ vs. $22.3 \pm 2.7 \mathrm{~g}, P<0.01)$. Upon administration of chalcone, food intake was reduced in all groups receiving the treatment, but while the effect was more pronounced for doses exceeding $2 \mathrm{mg} / \mathrm{kg}$, the three doses Ch8, Ch16 and Ch32 had nearly the same effect, a trend that was also observed for the chalcone effect on cholesterol and LDL. The fact that this effect is also observed in normal rats should be emphasized, since chalcone could be suggested to possess an appetite-suppressing effect, and thus be potentially effective in patients who would need weight loss.

\section{Water intake}

As a well-known consequence of becoming diabetic, water intake was observed to significantly increase in the diabetic control group (DO) compared with NDO (98.0 $\pm 11.3 \mathrm{ml} v$ s. $34.5 \pm 4.8 \mathrm{ml}, P<0.001)$ (Fig. 6). Chalcone administration decreased the amount of water taken by the diabetic groups, again with Ch8, Ch16 and Ch32 acting similarly and more strongly than Ch2. As expected, chalcone had no effect on the water intake in the non-diabetic groups.

\section{Urine volume}

In diabetic rats (DO), urine volume was significantly increased relative to the non-diabetic control (NDO) (72.6 $\pm 8.3 \mathrm{ml}$ vs. $17.3 \pm 2.7 \mathrm{ml}, P<0.001$ ) (Fig. 7), again 
(A)

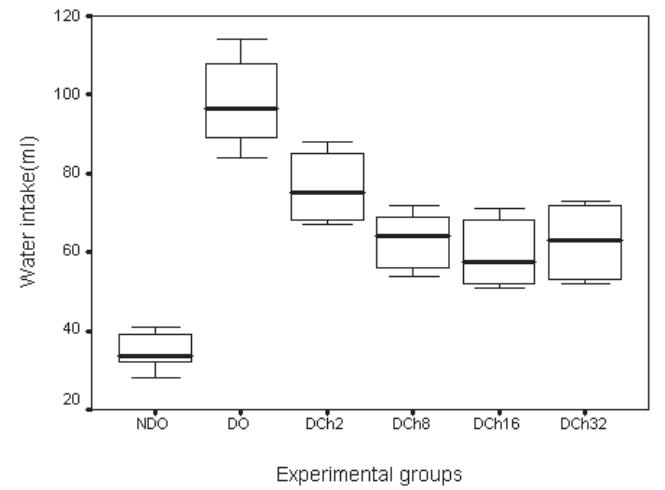

(B)

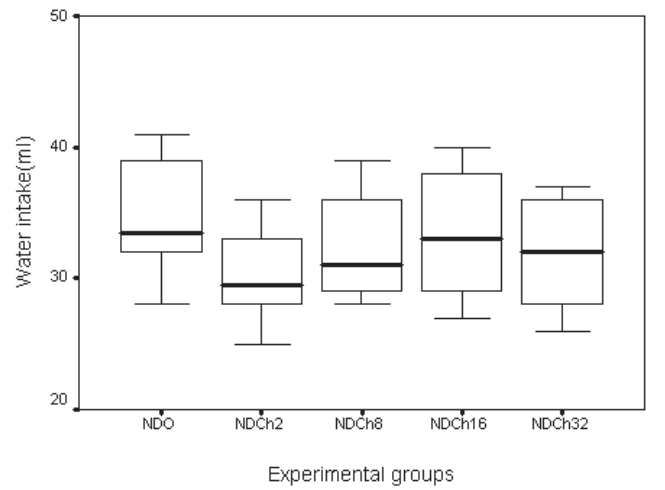

Figure 6. Effect of trans-chalcone treatment on water intake in diabetic control and non-diabetic rats

(A) Diabetic rats. Using one way ANOVA with Tukey-Kramer test at $P<0.05$ following results were found for the data: DO is significantly different from NDO; DCh2 is significantly different from NDO and DO; DCh8 is significantly different from NDO and DO; DCh16 is significantly different from NDO, DO and DCh2; Dch32 is significantly different from NDO and DO. (B) Non-diabetic rats. Water intake in control and experimental groups were found to have no significant difference using one way ANOVA with Tukey-Kramer test at $P<0.05$.

Table 2. Effect of chalcone treatment on serum lipids in non-diabetic rats.

Data are expressed as mean \pm S.D. for six rats. a, significantly different from non-diabetic control group (NDO) using one way ANOVA with Tukey-Kramer test at $P<0.05$.

\begin{tabular}{|c|c|c|c|c|c|}
\hline Serum level (mg/dl) & NDO & NDCh2 & NDCh8 & NDCh16 & NDCh32 \\
\hline Cholesterol & $85.5 \pm 11.5$ & $71.1 \pm 10.5^{a}$ & $70.6 \pm 9.4^{a}$ & $71.3 \pm 10.4^{a}$ & $72.6 \pm 8.1^{\mathrm{a}}$ \\
\hline Triglycerides & $63.5 \pm 8.4$ & $52.3 \pm 7.7^{a}$ & $51.6 \pm 6.5^{a}$ & $50.5 \pm 5.2^{\mathrm{a}}$ & $52.1 \pm 8.3^{a}$ \\
\hline VLDL-C & $12.7 \pm 1.7$ & $10.5 \pm 1.5^{\mathrm{a}}$ & $10.3 \pm 1.3^{a}$ & $10.1 \pm 1.1^{\mathrm{a}}$ & $10.4 \pm 1.6^{a}$ \\
\hline LDL-C & $22.5 \pm 4.6$ & $17.1 \pm 3.2^{\mathrm{a}}$ & $16.5 \pm 4.6^{a}$ & $17.3 \pm 4.3$ & $17.5 \pm 4.5$ \\
\hline HDL-C & $44.5 \pm 9.6$ & $43.6 \pm 8.6$ & $44.3 \pm 9.4$ & $45.1 \pm 7.2$ & $44.5 \pm 8.9$ \\
\hline
\end{tabular}

as a well-known consequence of diabetes. Administration of chalcone reduced urine volume in a dose-dependent manner, but without any significant difference between Ch16 and Ch32. This is the same trend observed in the effect of chalcone on blood glucose, which is directly related to the urine volume.

Again, no significant effect was detected on the urine volume of non-diabetic rats upon administration of chalcone.

(A)

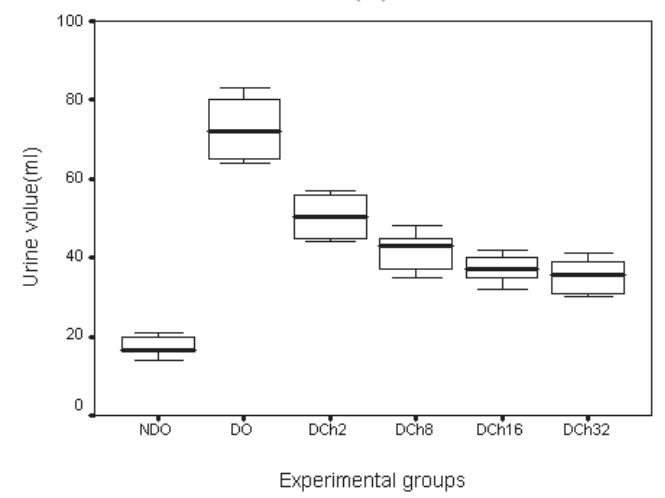

\section{Unwanted effect}

At the end of the experiment, the gastrointestinal tract of sacrificed animals was found to show anomalies in the groups treated with higher doses of chalcone (especially DCh32 and NDCh32). The gut and intestine were swollen and their walls got thinner, so that their content was visible from the outside. Chalcone is a yellow compound, and yellow aggregates were observed in the gut. This could be indicative of a bioavailability problem,

Figure 7. Effect of trans-chalcone treatment on urine volume in diabetic rats.

(A) Diabetic rats. Using one way ANOVA with Tukey-Kramer test at $P<0.0505$ following results were found for the data: DO is significantly different from NDO; DCh2 is significantly different from NDO and DO; DCh8 is significantly different from NDO and DO; DCh16 is significantly different from NDO, DO and DCh2; DCh32 is significantly different from NDO, DO and DCh2. (B) Non-diabetic rats. Urine volume in control and experimental groups have no significant difference using one way ANOVA with Tukey-Kramer test at $P<0.05$. 
which is suggestive of the need to design better-absorbable chalcone derivatives, or use of intermediate dosages.

\section{CONCLUSION}

trans-Chalcone was shown to possess $\alpha$-amylase inhibitory property in vivo, in a rat model of type 1 diabetes, and to be able to correct to a great extent the complications associated with the diabetic state. Significant reducing of hyperglycemia, as well as normalization of lipid profile were obtained when using a dose of $8 \mathrm{mg} /$ $\mathrm{kg}$, alongside with a reduction of water intake and urine volume. Weight loss and decrease of food intake, also observed in the case of a peptidic $\alpha$-amylase inhibitor from Phaseolus vulgaris (Tormo et al., 2004), which occur in both normal and diabetic rats, is another interesting result, and suggestive of the potential use of trans-chalcone as an effective agent for the treatment and even the prevention of obesity. Reduced blood glucose levels of normal rats is also another point of interest, as sharp increases of glucose levels in postprandial states are considered a risk factor in the transition from glucose intolerance toward diabetes, and metabolic syndrome complications among which cardiovascular problems are the most serious (Beisswenger et al., 2004; Chiasson et al., 2004; Yamagishi et al., 2005). The observed reduction of insulin levels could be suggestive of an insulinlike effect of the compound, or as stated in the case of acarbose, a possible secondary effect that would be the result of its main mechanism of action (Rosak \& Mertes, 2009). Overall, a comparison of the results obtained here with the ones reported about other $\alpha$-amylase inhibitors shows a good correlation; on the other hand, in reports of chalcone derivatives' effects, insulin mimetic properties and inhibition of TG secretion (Casaschi et al., 2004; Ogawa et al., 2005) are also mentioned, which are suggestive of a possibility for trans-chalcone to act on multiple targets, but this assumption needs further verification.

In conclusion, trans-chalcone is one of the few small molecules with $\alpha$-amylase inhibitory property that shows beneficial effect in the treatment of diabetes and related disorders, and could be proposed as a lead compound in this regard.

\section{Competing interests}

The authors declare that they have no competing interests.

\section{REFERENCES}

Alberton EH, Damazio RG, Cazarolli LH, Chiaradia LD, Leal PC, Nunes RJ, Yunes RA, Silva FR (2008) Influence of chalcone analogues on serum glucose levels in hyperglycemic rats. Chem Biol Interact 171: 355-362.

Aslan M, Orhan DD, Orhan N, Sezik E, Yesilada E (2007) A study of antidiabetic and antioxidant effects of Helichrysum graveolens capitulums in streptozotocin-induced diabetic rats. J Med Food 10: 396-400.

Bachorik PS (1997) Measurement of low-density lipoprotein cholesterol. In Handbook of lipoprotein testing. Rifai N, Warnick GR, Dominiczack MH, eds, pp 145-160. AACC Press, Washington.

Beisswenger P, Heine RJ, Leiter LA, Moses A, Tuomilehto J (2004) Prandial glucose regulation in the glucose triad: emerging evidence and insights. Endocrine 25: 195-202.

Boivin M, Flourie B, Rizza RA, Go VL, DiMagno EP (1988) Gastrointestinal and metabolic effects of amylase inhibition in diabetics. Gastroenterology 94: 387-394.
Casaschi A, Maiyoh GK, Rubio BK, Li RW, Adeli K, Theriault AG (2004) The chalcone xanthohumol inhibits triglyceride and apolipoprotein B secretion in HepG2 cells. J Nutr 134: 1340-1346.

Ceriello A (2005) Postprandial hyperglycemia and diabetes complications: is it time to treat? Diabetes 54: 1-7.

Chiasson JL, Josse RG, Gomis R, Hanefeld M, Karasik A, Laakso M (2004) Acarbose for the prevention of type 2 diabetes, hypertension and cardiovascular disease in subjects with impaired glucose tolerance: facts and interpretations concerning the critical analysis of the STOP-NIDDM Trial data. Diabetologia 47: 969-975.

Cole TG, Klotzsch SG, McNamara J (1997) Measurement of triglyceride concentration. In Handbook of lipoprotein testing. Rifai N, Warnick GR, Dominiczack MH, eds, pp 115-126. AACC Press, Washington.

Deeg R, Ziegenhorn J (1983) Kinetic enzymic method for automated determination of total cholesterol in serum. Clin Chem 29: 17981802.

Ferrer JL, Austin MB, Stewart CJr, Noel JP (2008) Structure and function of enzymes involved in the biosynthesis of phenylpropanoids. Plant Physiol Biochem 46: 356-370.

Friedewald WT, Levy RI, Fredrickson DS (1972) Estimation of the concentration of low-density lipoprotein cholesterol in plasma, without use of the preparative ultracentrifuge. Clin Chem 18: 499-502.

Godbout A, Chiasson JL (2007) Who should benefit from the use of $\alpha$-glucosidase inhibitors? Curr Diab Rep 7: 333-339.

Gosavi A, Flaker G, Gardner D (2006) Lipid management reduces cardiovascular complications in individuals with diabetes and prediabetes. Prev Cardiol 9: 102-107.

Hwang IK, Kim DW, Park JH, Lim SS, Yoo KY, Kwon DY, Moon WK, Won MH (2009) Effects of grape seed extract and its ethylacetate/ethanol fraction on blood glucose levels in a model of type 2 diabetes. Phytother Res 23: 1182-1185.

Kim JS, Kwon CS, Son KH (2000) Inhibition of $\alpha$-glucosidase and amylase by luteolin, a flavonoid. Biosci Biotechnol Biochem 64: 24582461

Knott RM, Grant G, Bardocz S, Pusztai A, de Carvalho AF, Hesketh JE (1992) Alterations in the level of insulin receptor and GLUT-4 mRNA in skeletal muscle from rats fed a kidney bean (Phaseolus vulgaris) diet. Int J Biochem 24: 897-902.

Kruse-Jarres JD, Kaiser C, Hafkenscheid JC, Hohenwallner W, Stein W, Bohner J, Klein G, Poppe W, Rauscher E (1989) Evaluation of a new $\alpha$-amylase assay using 4,6-ethylidene-(G7)1,4-nitrophenyl(G1)- $\alpha$-D-maltoheptaoside as substrate. J Clin Chem Clin Biochem 27: $103-113$.

Lankisch M, Layer P, Rizza RA, DiMagno EP (1998) Acute postprandial gastrointestinal and metabolic effects of wheat amylase inhibitor (WAI) in normal, obese, and diabetic humans. Pancreas 17: 176-181.

Lawrence JM, Liese AD, Liu L, Dabelea D, Anderson A, Imperatore G, Bell R (2008) Weight-loss practices and weight-related issues among youth with type 1 or type 2 diabetes. Diabetes Care 31: 2251-2257.

Li W, Dai RJ, Yu YH, Li L, Wu CM, Luan WW, Meng WW, Zhang XS, Deng YL (2007) Antihyperglycemic effect of Cephalotaxus sinensis leaves and GLUT-4 translocation facilitating activity of its flavonoid constituents. Biol Pharm Bull 30: 1123-1129.

Lo Piparo E, Scheib H, Frei N, Williamson G, Grigorov M, Chou CJ (2008) Flavonoids for controlling starch digestion: structural requirements for inhibiting human $\alpha$-amylase. J Med Chem 51: 3555-3561.

Machius M, Vertesy L, Huber R, Wiegand G (1996) Carbohydrate and protein-based inhibitors of porcine pancreatic $\alpha$-amylase: structure analysis and comparison of their binding characteristics. I Mol Biol 260: 409-421.

Najafian M, Ebrahim-Habibi A, Hezareh N, Yaghmaei P, Parivar K, Larijani B (2010) Trans-chalcone: a novel small molecule inhibitor of mammalian alpha-amylase. Mol Biol Rep E-pub DOI 10.1007/ s11033-010-0271-3.

Navas PB (2009) Chemical composition of the virgin oil obtained by mechanical pressing form several grape seed varieties (Vitis vinifera L.) with emphasis on minor constituents. Arch Latinoam Nutr 59: 214-219 (in Spanish).

Nijveldt RJ, van Nood E, van Hoorn DE, Boelens PG, van Norren K, van Leeuwen PA (2001) Flavonoids: a review of probable mechanisms of action and potential applications. Am J Clin Nutr 74: $418-425$.

Ogawa H, Ohno M, Baba K (2005) Hypotensive and lipid regulatory actions of 4-hydroxyderricin, a chalcone from Angelica keiskei, in stroke-prone spontaneously hypertensive rats. Clin Exp Pharmacol Physiol 32: 19-23.

Portha B, Levacher C, Picon L, Rosselin G (1974) Diabetogenic effect of streptozotocin in the rat during the perinatal period. Diabetes 23: 889-895.

Portha B, Blondel O, Serradas P, McEvoy R, Giroix MH, Kergoat M, Bailbe D (1989) The rat models of non-insulin dependent diabetes induced by neonatal streptozotocin. Diabetes Metab 15: 61-75.

Rebolledo OR, Actis Dato SM (2005) Postprandial hyperglycemia and hyperlipidemia-generated glycoxidative stress: its contribution to the 
pathogenesis of diabetes complications. Eur Rev Med Pharmacol Sci 9: 191-208.

Rosak C, Mertes G (2009) Effects of acarbose on proinsulin and insulin secretion and their potential significance for the intermediary metabolism and cardiovascular system. Curr Diabetes Rev 5: 157-164.

Sharma B, Balomajumder C, Roy P (2008) Hypoglycemic and hypolipidemic effects of flavonoid rich extract from Eugenia jambolana seeds on streptozotocin induced diabetic rats. Food Chem Toxicol 46: 2376-2383.

Tadera K, Minami Y, Takamatsu K, Matsuoka T (2006) Inhibition of $\alpha$-glucosidase and $\alpha$-amylase by flavonoids. J Nutr Sci Vitaminol (Tokyo) 52: 149-153.

Tangolar SG, Ozogul Y, Tangolar S, Torun A (2007) Evaluation of fatty acid profiles and mineral content of grape seed oil of some grape genotypes. Int J Food Sci Nutr 60: 1-8.

Tormo MA, Gil-Exojo I, Romero de Tejada A, Campillo JE (2004) Hypoglycaemic and anorexigenic activities of an $\alpha$-amylase inhibitor from white kidney beans (Phaseolus vulgaris) in Wistar rats. Br J Nutr 92: 785-790.
Verges B (2009) Lipid disorders in type 1 diabetes. Diabetes Metab 35: 353-360.

Verhoeyen ME, Bovy A, Collins G, Muir S, Robinson S, de Vos CH, Colliver S (2002) Increasing antioxidant levels in tomatoes through modification of the flavonoid biosynthetic pathway. J Exp Bot 53: 2099-2106.

Wiebe DA, Warnick GR (1997) Measurement of high-density lipoprotein cholesterol. In Handbook of lipoprotein testing. Rifai N, Warnick GR, Dominiczack MH, eds, pp 127-144. AACC Press, Washington.

Yamagishi S, Nakamura K, Takeuchi M (2005) Inhibition of postprandial hyperglycemia by acarbose is a promising therapeutic strategy for the treatment of patients with the metabolic syndrome. Med Hypotheses 65: 152-154.

Yoon SH, Robyt JF (2003) Study of the inhibition of four $\alpha$ amylases by acarbose and its $4 \mathrm{IV}-\alpha$-maltohexaosyl and $4 \mathrm{IV}-\alpha$-maltododecaosyl analogues. Carbohydr Res 338: 1969-1980. 